\title{
El año que nos volvimos insostenibles: Análisis de la producción española en Sustainability (2020)
}

\section{The year we became unsustainable: Analysis of Spanish production in Sustainability (2020)}

\author{
Rafael Repiso; Adoración Merino-Arribas; Álvaro Cabezas-Clavijo
}

Cómo citar este artículo:

Repiso, Rafael; Merino-Arribas, Adoración; Cabezas-Clavijo, Álvaro (2021). "El año que nos volvimos insostenibles: Análisis de la producción española en Sustainability (2020)". Profesional de la información, v. 30, n. 4, e300409.

https://doi.org/10.3145/epi.2021.jul.09

Artículo recibido el 01-06-2021

Aceptación definitiva: 05-07-2021

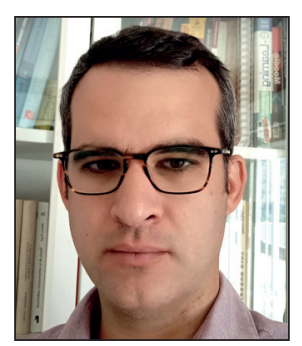

Rafael Repiso

https://orcid.org/0000-0002-2803-7505

Universidad Internacional de La Rioja

Av. de la Paz, 137

26006 Logroño, España

rafael.repiso@gmail.com

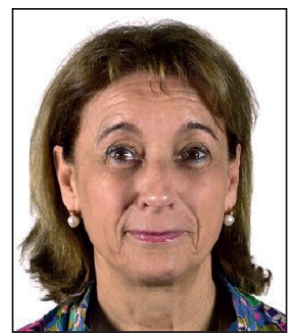

Adoración Merino-Arribas

https://orcid.org/0000-0002-3294-9996

Universidad Internacional de La Rioja

Av. de la Paz, 137

26006 Logroño, España

dory.merino@unir.net

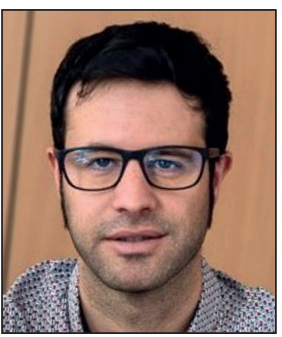

\author{
Álvaro Cabezas-Clavijo \\ https://orcid.org/0000-0001-9641-8855 \\ Universidad Internacional de La Rioja \\ Av. de la Paz, 137 \\ 26006 Logroño, España \\ alvaro.cabezas@unir.net
}

\section{Resumen}

La revista Sustainability, de la editorial MDPI, fue en 2020 la revista que publicó más trabajos de investigadores de instituciones españolas. Esto justifica un examen detallado de la producción científica española en dicha revista. Se analiza también la labor de edición de números monográficos en los cuales se han publicado trabajos con autores españoles. Para ello se ha identificado la pertenencia de cada trabajo a números especiales y a números regulares de la revista, identificando si el autor del trabajo es también editor del monográfico, o compañero de universidad. Igualmente se estudia la nacionalidad de los editores. Se analiza la temática de los artículos y su pertenencia al área de la revista. La mayor parte de los trabajos publicados por autores españoles se difundieron en números especiales (81,6\%), principalmente editados por autores de la misma nacionalidad $(63,1 \%)$. Se ve asimismo que existe una importante producción realizada por el mismo editor del monográfico, o por colegas de la misma institución. También se encuentra que un número sustancial de trabajos publicados en Sustainability (42,3\%) guarda una relación mínima o inexistente con la temática de la Sostenibilidad. Finalmente, se discuten las implicaciones de estos hallazgos a la luz de las estrategias de publicación de los investigadores españoles y de las políticas de evaluación de la ciencia aplicadas en España. Los principales aspectos polémicos identificados tienen que ver con la publicación de trabajos de otros temas, el valor de la publicación en monográficos que en algunos casos podrían ser considerados como endogamia y los reducidos tiempos de edición.

\section{Palabras clave}

Revistas científicas; Megajournals; Producción científica; Números monográficos; Números especiales; Costes de edición de artículos; Temática de la revista; Sustainability; MDPI.

\section{Abstract}

The journal Sustainability, published by MDPI, was the journal that published the most papers by researchers from Spanish institutions in 2020. This justifies a detailed examination of the Spanish scientific production in this journal. At the same 
time, the work of editing monographic issues in which papers with Spanish authors have been published is analyzed. For this purpose, we have identified whether each paper belongs to regular or to special issues of the journal, identifying whether the author of the paper is also editor of the monograph, or a university colleague. The nationality of the editors was also studied. The subject matter of the articles and their belonging to the area of the journal were analyzed. Most of the papers published by Spanish authors were published in special issues (81.6\%), mainly edited by authors of the same nationality (63.1\%). It is also found that there is an important production carried out by the same editor of the monograph, or by colleagues from the same institution. It is also found that a substantial number of works published in Sustainability (42.3\%) have minimal or no relationship with the subject of sustainability. Finally, the implications of these findings are discussed under the light of the publication strategies of Spanish researchers and the science evaluation policies applied in Spain. The main controversial aspects identified have to do with the publication of papers on other subjects, the value of publishing in monographs, which in some cases could be considered as inbreeding, and the short editing times.

\section{Keywords}

Scientific journals; Megajournals; Scientific production; Special issues; Monograph issues; Inbreeding; Article processing charges; APCs; Journal scope; Sustainability; MDPI.

Financiación

Trabajo financiado por el Vicerrectorado de Investigación de la Universidad Internacional de La Rioja.

Agradecimientos

Nuestro agradecimiento a los revisores, por su aportación al trabajo y celeridad.

\section{Introducción}

El año 2020 va a recordarse en la historia de la investigación española como el año que nos "convertimos" en investigadores sostenibles, o insostenibles según se mire. Una revista, Sustainability, de una editorial con origen chino-suizo Multidisciplinary Digital Publishing Institute (MDPI) publicaba la cifra récord de 1.417 trabajos de españoles, seguida muy de cerca por otra revista de la misma editorial y temática similar, International journal of environmental research and public health con 1.414 trabajos. Este fenómeno de un tan alto número de trabajos se sitúa como un hito en la historia de la investigación española y justifica que se estudie en profundidad, pues en volumen excede a la producción española en muchas áreas. Pensemos que de las 152 categorías temáticas de Web of Science en las que han publicado los investigadores españoles en 2020, sólo en 29 se superan estas cifras de publicación, es decir lo publicado en una sola revista excede lo publicado por investigadores españoles en el mismo año en 123 categorías científicas.

La revista Sustainability es la revista mundial que publicó más trabajos de autores con afiliación española en 2020, lo que nos lleva a preguntarnos:

¿Cómo se ha erigido una revista del área de Ciencias Medioambientales en la principal difusora de la investigación española?

¿Qué estrategias sigue esta revista que la hace atractiva para los autores españoles? ¿Es sostenible seguir publicando de este modo en Sustainability?

\subsection{Los megajournals}

La pujanza de revistas como la analizada en este trabajo evidencia el modelo de publicación de los denominados megajournals. Se trata principalmente de revistas:

- con producción abundante;

- que realizan un peer review que valora únicamente la solvencia científica del trabajo, frente a la complejidad, utilidad, originalidad o interés del mismo (Björk; Catani, 2016);

- que ofrecen acceso abierto completo a la publicación mediante el modelo article processing charge (APC);

- que son de naturaleza multidisciplinar, ya que publican trabajos en varias áreas del conocimiento (Spezi et al., 2017).

Otras características asociadas a este modelo de revistas son:

- cuentan en general con tasas de aceptación más altas que las revistas disciplinares;

- ofrecen una publicación más rápida que las revistas tradicionales;

- muchas veces ofrecen una vía de publicación a artículos que han sido rechazados por otras revistas más selectivas de la misma casa editorial (Björk, 2015).

En los últimos años son numerosas las mega-revistas que han surgido, de la mano de varios editores. Entre otras:

- PLoS one (de Public Library of Science, en 2006);

- Scientific reports (de Nature Publishing Group, en 2011);

- Sage open (de Sage, en 2011);

- IEEE access (de IEEE, en 2013). 
A estas mega-revistas de editoriales consolidadas se han unido otras revistas de editoriales como MDPI o Frontiers, que, aunque en apariencia son revistas disciplinares, en la práctica publican masivamente artículos de una gran variedad temática, lo que las convierte también en megajournals. Según datos de Web of Science, en 2020 las revistas que publicaron más trabajos a nivel mundial fueron Scientific reports (21.158), IEEE access (17.511), PloS one (15.574) y en cuarto lugar Sustainability (10.577). Los artículos españoles representan entre el 3,5\% y el 5\% de los artículos de las primeras; en Sustainability su presencia asciende al $13 \%$.

En lo que respecta a la producción científica española, desde 2011 la revista donde se han publicado más artículos de autores españoles ha sido siempre un megajournal. Entre 2011 y 2016, así como en 2018 PLoS one fue el principal medio que difundió la ciencia realizada por instituciones españolas, mientras que en 2017 y 2019 fue Scientific reports. El año 2020 marca un cambio de tendencia, ya que por primera vez son dos revistas de la editorial MDPI las que publican más trabajos con afiliación española, en concreto la revista Sustainability, con 1.417 trabajos publicados, seguida a muy corta distancia por International journal of environmental research and public health (IJERPH) con 1.414 artículos, ambas del grupo editorial MDPI.

Tabla 1. Revistas donde España publica un mayor número de trabajos (2011-2020)

\begin{tabular}{|l|l|l|}
\hline Año & \multicolumn{1}{|c|}{ Primera } & \multicolumn{1}{|c|}{ Segunda } \\
\hline 2020 & Sustainability (1.417) & International journal of environmental research and public health (1.414) \\
\hline 2019 & Scientific reports (1.024) & PLoS one (795) \\
\hline 2018 & PLoS one (911) & Scientific reports (804) \\
\hline 2017 & Scientific reports (1.011) & PLoS one (928) \\
\hline 2016 & PLoS one (981) & Scientific reports (688) \\
\hline 2015 & PLoS one (1.092) & Astronomy astrophysics (408) \\
\hline 2014 & PLoS one (1.191) & Astronomy astrophysics (448) \\
\hline 2013 & PLoS one (1.206) & Astronomy astrophysics (376) \\
\hline 2012 & PLoS one (1.032) & Astronomy astrophysics (391) \\
\hline 2011 & PLoS one (626) & Astronomy astrophysics (364) \\
\hline
\end{tabular}

Fuente: Elaborado a partir de datos de Web of Science (Core Collection)

\subsection{Números especiales monográficos}

Una de las características distintivas de la revista Sustainability es la edición de abundantes números especiales o special issues. Los números especiales, temáticos o monográficos son un aspecto poco analizado de la comunicación científica, si bien es una práctica habitual en las revistas académicas. La edición de números temáticos se relaciona con diversos aspectos relativos al poder e influencia de una revista en su disciplina. En particular se puede vincular con el desarrollo y difusión del conocimiento en una disciplina concreta (Olk; Griffith, 2004). Si una revista es capaz de proponer números especiales sobre temas atractivos, frentes de investigación emergentes o nichos en la especialidad que generen atención en la comunidad, esos monográficos pueden alcanzar mayor impacto que los números regulares de la misma publicación. Si, por el contrario, los números especiales tratan temas de escaso interés, o excesivamente especializados, existe el riesgo de que la revista reciba manuscritos de menor calidad, reduciendo la influencia de la publicación (Conlon et al., 2006).

Diversos estudios han puesto de manifiesto la creciente importancia de los números temáticos, en campos como el Management, la Psicología o la Comunicación. Khan et al. (2021) señalan que las principales revistas de Management pasaron de publicar un 2\% de artículos en números especiales en 1984 al 10\% en 2015. En un estudio previo (Olk; Griffith, 2004) también se constata el aumento en el número de special issues en las principales revistas de Management. Por su parte González-Sala et al. (2017) encuentran que el 25,5\% de artículos en diez revistas iberoamericanas de Psicología se publican en números especiales mientras que Repiso et al. (2021) concluyen que hasta el 19\% de los artículos en las revistas de Comunicación en el período 2015-2019 se publican en números temáticos.

En el estudio con la muestra más amplia (casi 3 millones de artículos publicados en más de 200.000 números de 4.559 revistas) realizado hasta el momento (Sainte-Marie; Mongeon; Larivière, 2020) se pone de manifiesto que la importancia de los números temáticos es mayor en Artes y Humanidades (casi un $24 \%$ del total de números publicados), y en Ciencias Sociales $(13,7 \%)$ que en el resto de las disciplinas. Para el conjunto de las ciencias, uno de cada diez números de revista son especiales o monográficos.

Otros estudios que comparan números monográficos y números regulares se han centrado en el tiempo de aceptación de los trabajos (Olk; Griffith, 2004; González-Sala et al., 2017), o en la citación que reciben los artículos publicados en 
números especiales en comparación con la recibida por los trabajos editados en números regulares de la misma revista (Conlon et al., 2006; González-Sala et al., 2017; Gardner et al., 2010; Khan; Ali; Ashraf, 2020; Khan et al., 2021; Sainte-Marie; Mongeon; Larivière, 2020; Repiso et al., 2021).

Un asunto menos tratado en la bibliografía sobre volúmenes monográficos ha sido el papel del editor o editores invitados de los números especiales. Repiso et al. (2021) señalan que lo habitual es que estén subordinados al editor de la revista y se integren en los procesos evaluativos de la misma, pero en algunos casos sustituyen al editor general en el contexto de su número temático, controlando el proceso de evaluación, es decir, proponiendo temáticas concretas, designando revisores y lo que es más importante, decidiendo qué trabajos se publican y cuáles se rechazan. Las motivaciones de los editores invitados para proponer o participar en un monográfico varían desde el mero servicio a la comunidad, el ejercer control u orientar las temáticas y publicaciones en una materia (Mowday, 2006), situarse como referente en ese tema emergente, contar con mayor facilidad para publicar en dicho número especial, o cumplir con los requisitos impuestos en los procesos de promoción y recompensa académica. No en vano ejercer como editor invitado en volúmenes especiales es un mérito reconocido en los criterios de la principal agencia española de evaluación en algunas áreas como Ciencias Sociales para las figuras de Profesor Titular y Catedrático de Universidad (Aneca, 2019).

\subsection{La revista Sustainability}

Sustainability es una de las 338 revistas publicadas por la editorial suiza $M D P I^{1}$. Se inició en 2009 con periodicidad trimestral, pasando a una frecuencia mensual en 2010, y a periodicidad quincenal en 2019. Realmente funciona como una revista de publicación continua, es decir, a medida que los artículos se aceptan, se van publicando online, acortando los plazos entre aceptación del manuscrito y publicación.

Se trata de una revista en acceso abierto, dentro de la denominada ruta dorada, en la cual los costes son asumidos por los autores mediante APC (article processing charges), o tasa por procesamiento del artículo. El coste estándar es de 1.900 francos suizos por artículo (1.720 euros aproximadamente ${ }^{2}$ ), si bien la editorial ofrece descuentos a través de programas para instituciones afiliadas, para autores de países subdesarrollados y en vías de desarrollo, así como para revisores especialmente activos. Hay que indicar que esta editorial modifica el coste de publicación en relación directa al posicionamiento de sus revistas en Web of Science o Scopus.

Tabla 2. Posicionamiento en cuartiles de la revista Sustainability en Web of Science y Scopus (2010-2019)

\begin{tabular}{|c|c|c|c|c|}
\cline { 2 - 5 } \multicolumn{1}{c|}{} & \multicolumn{2}{c|}{$\begin{array}{c}\text { Journal Citation Reports } \\
\text { (JCR) }\end{array}$} & \multicolumn{2}{c|}{$\begin{array}{c}\text { SCImago Journal Rank } \\
\text { (SJR) }\end{array}$} \\
\hline Año & FI & Q & SJR & Q \\
\hline 2010 & & & 0.152 & Q3 \\
\hline 2011 & & & 0.263 & Q2 \\
\hline 2012 & & & 0.463 & Q2 \\
\hline 2013 & 1.077 & Q3 & 0.521 & Q2 \\
\hline 2014 & 0.942 & Q3 & 0.501 & Q2 \\
\hline 2015 & 1.343 & Q3 & 0.482 & Q2 \\
\hline 2016 & 1.789 & Q2 & 0.548 & Q2 \\
\hline 2017 & 2.075 & Q2 & 0.537 & Q2 \\
\hline 2018 & 2.592 & Q2 & 0.549 & Q2 \\
\hline 2019 & 2.576 & Q2 & 0.581 & Q2 \\
\hline
\end{tabular}

Fuente: Elaborado a partir de los datos de Journal Citation Reports y de SCImago Journal Rank. Nota: Cuando la revista se sitúa en dos cuartiles distintos en el mismo año, se toma el cuartil superior.

Esta revista recibió su primer factor de impacto en Journal Citation Reports (JCR) en 2013, posicionándose en tercer cuartil (Q3) y ascendiendo posteriormente al segundo (Q2), posición que ha ocupado desde el año 2016 a 2019 (último disponible). La revista se indexa en cuatro disciplinas:

- dos de Science Citation Index Expanded (SCIE): "Green \& Sustainable Science \& Technology" y "Environmental Sciences"; - dos de Social Sciences Citation Index (SSCI): "Green \& Sustainable Science \& Technology" y "Environmental Studies".

Los datos tomados de SCImago Journal Rank describen una tendencia similar. Recibe su primer indicador SJR en 2010, situándose en Q3, y ya desde 2011 ocupa posiciones de manera ininterrumpida en Q2 en diversas categorías. Con datos de 2019, esta revista se clasifica en cinco categorías, situándose en todas ellas en segundo cuartil:

- "Energy Engineering and Power Technology";

- "Renewable Energy, Sustainability and the Environment";

- "Environmental Science (miscellaneous)";

- "Management, Monitoring, Policy and Law";

- "Geography, Planning and Development".

Varias son las características que hacen que esta revista sea atractiva a efectos de publicación y edición del monográfico:

- Su indexación en segundo cuartil en $J C R$ y en $S J R$, lo que genera que sea una revista válida en la mayoría de los campos científicos a efectos de valoración curricular en convocatorias como los procesos de acreditación del profesorado universitario o el reconocimiento de sexenios de investigación. La edición de números especiales en revistas de impacto también es un criterio de valoración en el proceso de acreditación curricular en España.

- Su velocísimo proceso de revisión y publicación. Según declara la propia revista, apenas 15 días después del envío del manuscrito, los autores reciben ya una primera decisión acerca de la aceptación o no del manuscrito. El tiempo medio entre la aceptación definitiva y la publicación es apenas de 3 días 3 . 
- Su política editorial respecto a los números especiales o monográficos. Ésta se caracteriza porque cualquier investigador es aceptado para dirigir un monográfico y no existen límites para ello. La propia editorial invita a autores a dirigir monográficos de áreas fuera de sus capacidades (Crosetto, 2021). Hay que tener en cuenta que los editores invitados toman decisiones acerca de la aceptación o no de los trabajos en los números que coordinan ${ }^{4}$.

- La transversalidad de la temática Sustainability donde puede ser entendido que cualquier elemento que se estudie puede contener "trazas" de sostenibilidad.

- Las campañas de descuentos y gratificaciones, que se basan en la existencia de convenios con universidades, lo que conlleva descuentos en los costes de publicación. Los revisores especialmente activos también reciben "tickets descuento", que les permiten publicar en condiciones más económicas.

Por úúltimo, hay que añadir que la editorial MDPI no es ajena a la polémica. Esta compañía fue incluida en 2014 en el listado de revistas "depredadoras" de Jeffrey Beall, para ser retirada un año después, como lo fue la editorial Frontiers. Siler (2020) sitúa a ambas editoriales en la zona gris, en la frontera de lo que puede considerarse como prácticas depredadoras (listados negros) y las editoriales honradas (listados blancos). De hecho, el propio Beall (2017) señala las presiones de MDPI como uno de los factores que le llevaron a cerrar dicho listado. Otro asunto similar fue la denuncia de diez miembros del equipo editorial de la revista Nutrients que dejaron sus cargos denunciando las imposiciones de $M D P I$ para aumentar la tasa de aceptación de manuscritos (De-Vrieze, 2018). Otros autores ponen el foco en la baja calidad en los procesos de revisión (Gjengedal, 2019), lo que permite aumentar la aceptación de manuscritos y, por ende, los beneficios derivados.

\subsubsection{Política editorial de $M D P I$ respecto a números especiales}

Sustainability fomenta activamente la publicación de números especiales sobre temas muy concretos. A modo de ejemplo señalamos en el cuadro 1 algunos títulos de números monográficos publicados en 2020.

Cuadro 1. Ejemplos de títulos de números especiales editados en Sustainability en 2020

3D Printing applications and sustainable construction

Academic motivation, performance and student well-being

Active aging: Educational innovations in the elderly

Biomass for sustainability: Resource, technology conversion and energy management

Business analytics and data mining for business sustainability

Citizen science and the role in sustainable development

Communication on sustainability in universities: A bridge between academia and society

Control methods to improve the sustainability and energy efficiency of $5 \mathrm{G}$ and optical networks.

Digital teaching competences for sustainable development

Entrepreneurship and the sustainable development goals for the business-health relationship

Historical ecology and landscape archaeology: Cross-disciplinary approaches to the long anthropocene

Dichos monográficos cuentan con uno o varios editores invitados (o autoinvitados, ya que un investigador puede proponerse para editar un número especial), y que supervisan todo el proceso editorial. La propia MDPI anima a que se realicen propuestas temáticas concretas de los autores, y que estos sean editados en colaboración entre autores de varias instituciones, con el objetivo de que se alcance una audiencia más amplia y de que se reduzcan los conflictos de intereses que pudiera haber entre editores y autores del número especial. Según la normativa de la editorial, los investigadores que deseen ejercer como editores invitados deben proponer incluso un listado de autores potenciales que podrían publicar en dicho número temático. Igualmente, se espera que difundan activamente el call for papers del número especial y que inviten a colegas de su área de conocimiento para que envíen manuscritos orientados a dicho número temático. Otro aspecto fundamental es que el editor del special issue publica gratuitamente en él. También normalmente tiene la posibilidad de ofrecer la publicación sin coste alguno a otros colegas, siempre y cuando dichas publicaciones superen el proceso de peer review; aunque no es oficial, es común en las revistas del grupo MDPI y uno de los privilegios de los editores (MDPI, 2018).

La revista declara que todos los manuscritos enviados a números temáticos son evaluados por pares, y que los editores deben evitar conflictos de intereses en la evaluación de artículos de autores con los que colaboran estrechamente o de su propia institución. La aprobación definitiva de un artículo se decide por parte de la oficina editorial, y por el Consejo Editorial de la revista, si bien, como se ha señalado previamente, los editores invitados normalmente toman decisiones acerca de los manuscritos recibidos para publicación dentro de su número monográfico ${ }^{4}$ así como sobre qué trabajos son elegibles para su publicación de Sustainability ha mostrado un crecimiento exponencial desde su indexación en Web of Science en el año 2011 forma gratuita. 


\subsection{Objetivos}

Debido a la importancia que la revista Sustainability ha adquirido en la difusión de la investigación realizada desde las instituciones españolas, se ha considerado como objetivo principal de este trabajo la caracterización de la actividad española en esta revista en el año 2020. Para ello se han considerado dos dimensiones:

- la publicación de artículos;

- la actividad en la edición de números monográficos o especiales con alguna publicación española.

Además, como objetivos específicos (OE) de este estudio, se señalan los siguientes:

OE1: Analizar las características de los special issues, a partir del editor/editores del mismo, y determinando la presencia de trabajos publicados por los propios editores, por investigadores del mismo centro de trabajo que los editores o por investigadores de la misma nacionalidad.

OE2: Determinar la temática de los artículos con firma española publicados en la revista, así como su vinculación con la materia de Sostenibilidad.

OE3: Estudiar los tiempos de publicación de los trabajos españoles según su pertenencia a algunos de los subgrupos estudiados (artículos en números regulares, artículos en monográficos, artículos en monográficos editados por autores españoles, artículos en monográficos editados por autores de la misma institución que el autor).

\section{Metodología}

El presente trabajo es un estudio bibliométrico descriptivo en el que se analiza la presencia y características de los trabajos elaborados por investigadores pertenecientes a alguna institución española publicados en la revista Sustainability en el año 2020.

La identificación y descarga de los trabajos se realizó en el mes de marzo (2021) utilizando Web of Science y limitando los documentos a artículos y revisiones donde al menos uno de los firmantes trabajaba en un centro español. Posteriormente, a través de la web de la revista Sustainability se identificó la pertenencia de cada artículo bien a un número especial o a un número ordinario. En el caso de los monográficos se identificó a sus editores, así como sus centros de trabajo. Además, se han realizado análisis generales para contextualizar los resultados, como es la producción de la revista Sustainability desde su indexación en Web of Science.

Una posterior revisión en mayo de 2021 ha permitido identificar que la producción de Sustainability indexada en Web of Science había descendido ligeramente (de 1.417 a 1.395 trabajos), debido a modificaciones de la base de datos. A efectos de transparencia, se ofrece como dato complementario el dataset analizado en este trabajo, que consta de los 1.417 artículos y revisiones firmados por instituciones españolas y publicados en Sustainability en 2020, según los datos de Web of Science a marzo de 2021.

Concretamente se ha analizado:

- Distribución de trabajos científicos en Sustainability desde su indexación en Web of Science (2011-2020). Se identifica la aportación española a lo largo de este período (figura 1).

- Distribución de los trabajos publicados por españoles según su pertenencia a monográficos o no, diferenciando si los monográficos son editados por investigadores españoles o de otros países. Se visualiza mediante un TreeMap realizado con Tableau (Murray; Chabot, 2013) (figura 2).

- Distribución de los artículos de investigadores españoles publicados en monográficos analizando la aportación de los propios editores al monográfico, de los compañeros de institución de los editores, así como de profesores de centros diferentes. Se visualiza mediante un TreeMap realizado con Tableau (figura 3).

- Pertenencia de los artículos al scope de la revista e identificación de los principales temas de los trabajos ajenos a la temática Sostenibilidad (figuras 4 y 5). Se han categorizado todos los trabajos estudiados (1.417), identificando si trataban sobre Sostenibilidad o no. Este procedimiento ha sido realizado de forma independiente por el primer y tercer autor del trabajo, obteniéndose un coeficiente de concordancia entre evaluadores (Kappa de Cohen) de 0,917, lo que equivale a una tasa de acuerdo muy elevada. En los casos en que hubo discrepancia, se llegó a un acuerdo entre los evaluadores, tras una revisión más detallada de los trabajos dudosos. Hay que indicar que los autores analizaron también la temática de los 127 trabajos publicados en el año 2011 por la revista Sustainability (primer año en Web of Science) como grupo control y para validar la percepción temática sobre Sostenibilidad; en este caso, sólo se detectó un trabajo $(0,8 \%)$ que no guardaba relación con el alcance temático declarado de la revista.

- Distribución de los tiempos de publicación de los artículos (desde su envío) según el grupo al que pertenezcan; números regulares, monográficos, monográficos dirigidos por españoles, monográficos dirigidos por extranjeros, monográficos dirigidos por compañeros de universidad, monográficos dirigidos por los autores del trabajo, artículos sobre sostenibilidad, artículos sobre otras temáticas. Se realiza un análisis de varianza (Anova) para determinar si existen diferencias estadísticamente significativas entre los grupos. Se usa XIstat (Addinsoft, 2019) para comprobar la significatividad. La distribución de los tiempos por grupo se visualiza mediante un boxplot elaborado con Tableau (figura 6). 
La distribución de tareas entre los autores fue:

Autor 1: Diseño, normalización de la carga de datos, análisis de los datos y redacción.

Autor 2: Carga de datos, identificación de la pertenencia a monográficos, adaptación del trabajo a las normas de la revista.

Autor 3: Análisis temático de artículos, redacción y revisión del trabajo.

\section{Resultados}

Sustainability ha mostrado un crecimiento exponencial desde su indexación en Web of Science en 2011. De la misma forma, la presencia de la academia española ha tenido un aumento muy significativo, al pasar de publicar el 0,02\% de los trabajos en 2011 ( 3 artículos) a ser protagonista de 1.417 trabajos en 2020 $(13,4 \%)$, lo que ha convertido a España en el tercer país con más artículos en dicha revista en 2020, por detrás de China y Estados Unidos, y a la Universidad de Granada como la tercera institución mundial con más trabajos (96). En los 1.417 trabajos analizados encontramos que hay 636 autores que publican dos o más trabajos; incluso hay un autor español que consigue publicar 14 trabajos, y otro un total de 10 trabajos en sólo 12 meses.

De los 1.417 artículos publicados por instituciones españolas en el año 2020 el $81,6 \%$ se ha publicado en algún número monográfico (1.156 artículos) y solamente el $18,4 \%$ lo ha hecho en números regulares (figura 2). De estos trabajos publicados en monográficos un porcentaje significativo, el 63,1\% (729 artículos, lo que representa el $51 \%$ del total), pertenece a su vez a monográficos cuyos directores trabajan en centros españoles (se han considerado en este grupo los trabajos en monográficos coeditados por autores españoles y extranjeros). Asimismo, se publican 427 trabajos en monográficos de editores exclusivamente extranjeros, lo que representa el $36,9 \%$ de los trabajos publicados en monográficos. Hay que indicar que, aunque el período de análisis es de un sólo año encontramos a 74 editores españoles que son protagonistas de dos o más monográficos, incluso

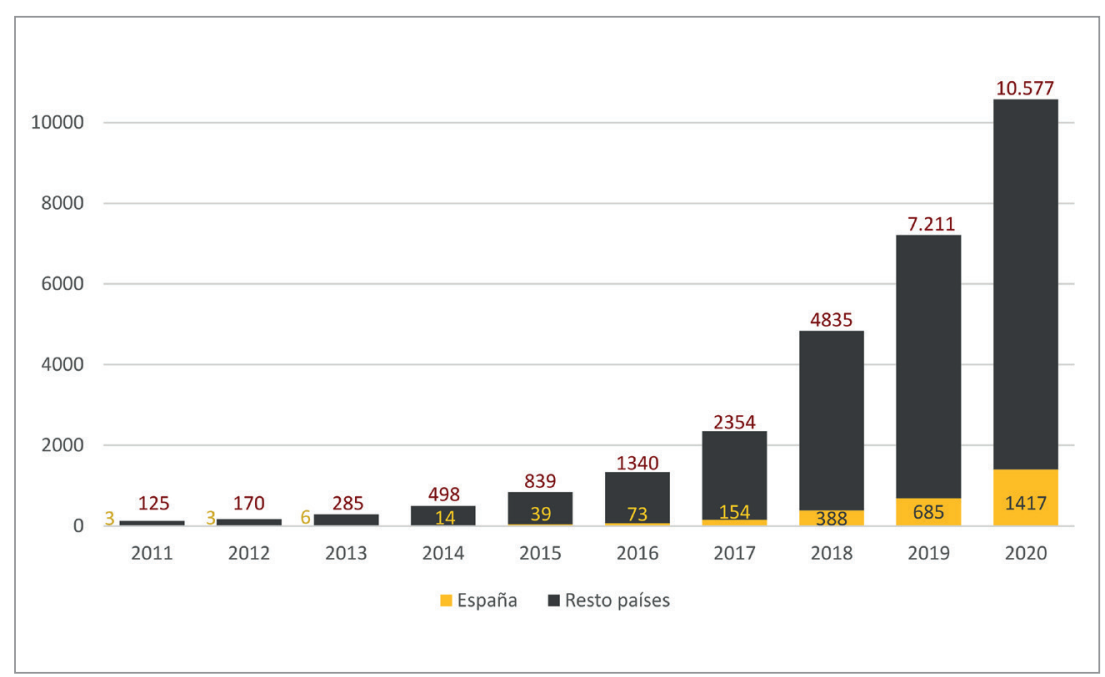

Figura 1. Evolución de las publicaciones de la revista Sustainability desde su indexación en Web of Science.

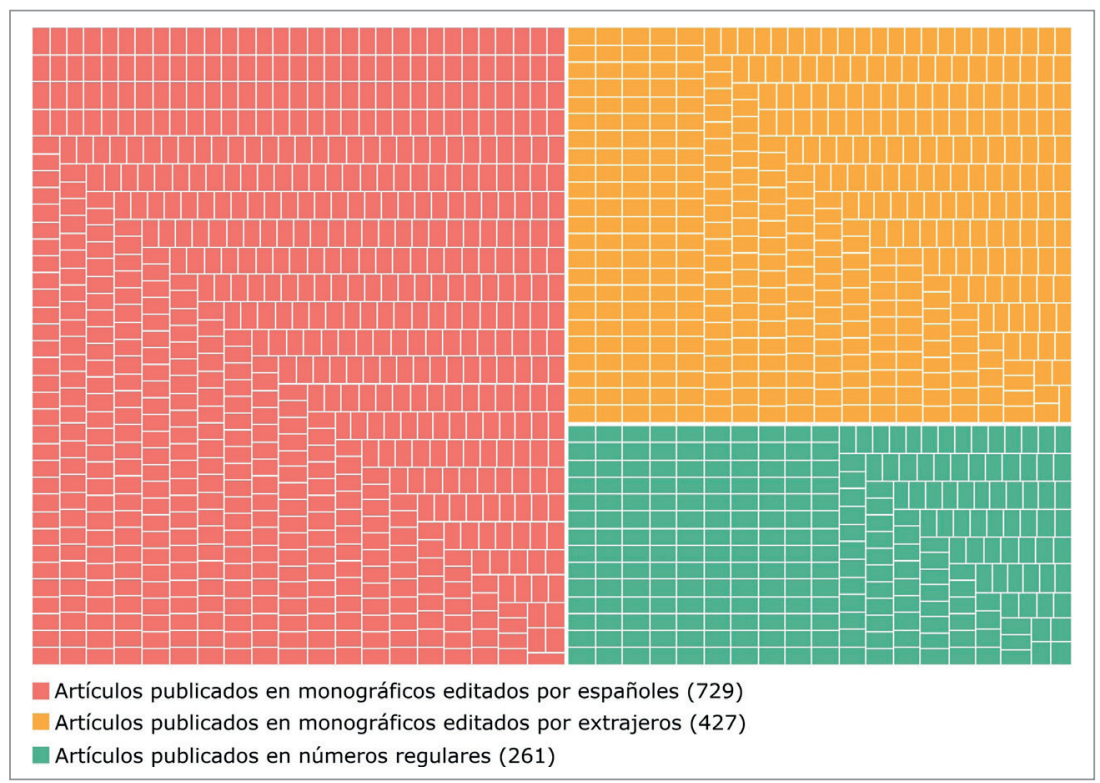

Figura 2. Distribución de los trabajos de investigadores españoles en Sustainability según publicación en número regular, en número especial editado por españoles, o en número especial editado por extranjeros

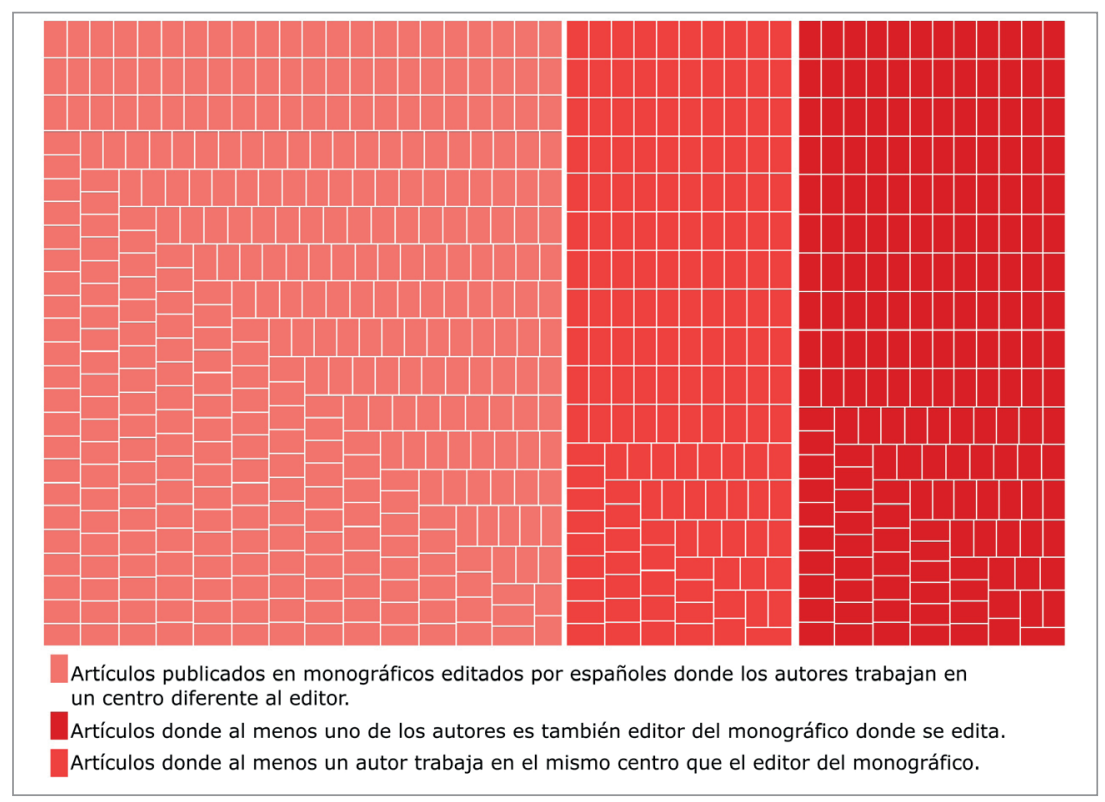

Figura 3. Distribución de los artículos de autores españoles publicados en monográficos editados por españoles en Sustainability (2020) 
hay tres editores que en un sólo año dirigen cuatro de estos números especiales.

Estos 1.156 trabajos publicados en 2020 en números especiales fueron publicados concretamente en 539 números temáticos distintos, de los cuales 265 contaron con editores invitados afiliados a alguna institución española, es decir, en prácticamente la mitad de los números temáticos donde publicaron autores españoles, el editor o coeditor de este era también un investigador español, si bien representan el $63 \%$ de los trabajos publicados en monográficos.

Si estudiamos los trabajos de españoles publicados bajo monográficos editados por españoles (729 trabajos) encontramos que 194 trabajos son autoría de los propios editores de los monográficos $(26,6 \%)$; a esto hay que sumarle 163 trabajos $(22,3 \%)$ que son editados por colegas de universidad de los editores de los monográficos. En conjunto, algo más de la mitad de los trabajos $(51,1 \%)$ provienen de autores de centros españoles distintos a los de los editores; la otra mitad, por el contrario $(48,9 \%)$ son artículos cuyos autores son editores o colegas de universidad de los editores del número especial.

Otro aspecto a destacar es la temática de los artículos publicados por España en Sustainability. En concreto, se han identificado 600 artículos (42,3\%) que abiertamente no guardan relación con la temática de la revista (Sostenibilidad y Medio Ambiente). Es en los números monográficos donde se publican más trabajos que no están relacionados con la temática central de la revista (45\%), mientras que en los números regulares es menor su presencia, significando el $28,3 \%$ de los trabajos (figura 4).

Las áreas donde más artículos de españoles sobre temas ajenos a Sostenibilidad se publican son Educación, Ciencias Empresariales, Deporte y Turismo (figura 5). Hay que indicar que de estos temas existen muchos otros trabajos de naturaleza híbrida que sí se han considerado dentro del scope de la revista, ya que tratan aspectos sobre sostenibilidad como pueden ser Turismo Verde, Enseñanza de la Sostenibilidad o Empresas Ecológicas.

Uno de los aspectos más destacados de la revista Sustainability es la agilidad de sus procesos de revisión. En promedio, los artículos de autores españoles publicados en el año tardan 39 días desde que son enviados hasta que se publican, si

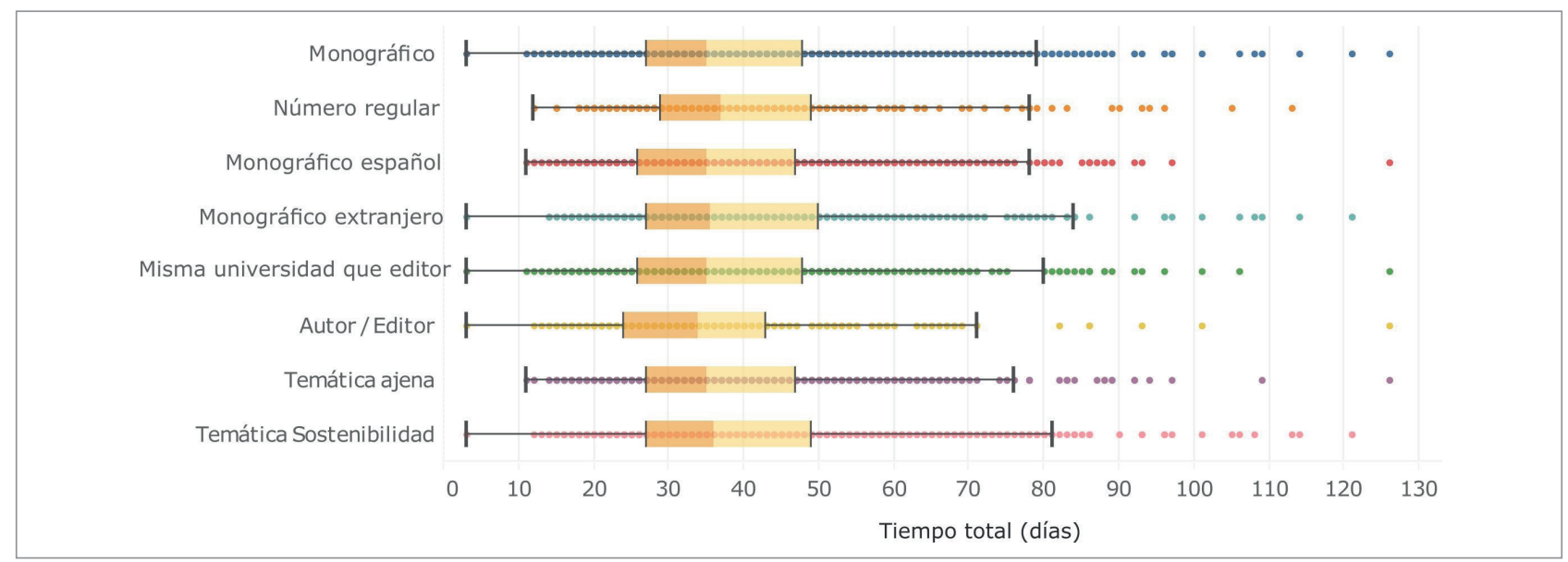

Figura 6. Distribución de tiempos de publicación de artículos españoles en Sustainability desde su envío según tipo 
bien estos tiempos se reducen ligeramente cuando el autor del artículo es el propio editor (36 días), o un colega del editor (38 días) (figura 6). De la misma forma existe una pequeña diferencia entre los tiempos si los artículos son publicados en números regulares (ligeramente más lentos) (40 días) o números monográficos (39 días).

Aunque los tiempos son muy similares, el análisis de la varianza (Anova) encuentra que existe significatividad entre las distribuciones de estos grupos (tabla 3).

\section{Discusión y conclusiones}

\subsection{Discusión}

En este estudio hemos caracterizado la actividad española en la revista Sustainability contemplando las dimensiones de producción científica, y edición de números especiales. En este sentido, en 2020 se detectaron 1.417 artículos publicados por autores españoles, lo que eleva la factura teórica del conjunto de trabajos a 2.458.367€, si bien esta cantidad habría que reducirla, debido a los descuentos y publicación gratuita con los que cuentan algunos autores. Esta revista no sólo representa el medio de comunicación científica donde más trabajos sobre Sostenibilidad publican los autores españoles; Sustainability tiene más producción española en algunas disciplinas, como Educación, Turismo o Deporte que cualquier revista especializada de dichas áreas.

Por ejemplo, en el área de Educación, sin contar los trabajos sobre Sostenibilidad y Educación (que son mayoría) identificamos 208 trabajos seguida muy de lejos por

- Revista de Educación con 32 artículos,

- Educación XX1 (27);

- Comunicar (27).

Lo mismo sucede en Ciencias del Deporte, donde Sustainability se sitúa como la revista que más trabajos de Deporte de autores españoles acoge, seguida por

- Journal of strength and conditioning research (55);

- Revista internacional de medicina y ciencias de la actividad física y del deporte (42).

E igual sucede en Turismo donde con 62 trabajos Sustainability se posicionaría como la revista con más trabajos del área seguida de

- Current issues in tourism (39);

- Tourism economics (30).

Sustainability está operando como una mega-revista multidisciplinar afectando a la producción científica española en varias áreas.

Se ha determinado que la parte más sustancial de la producción española en Sustainability $(81,6 \%)$ se vehicula a través de números especiales, editados, en su mayor parte por investigadores de instituciones españolas (63\%). Este es el perfil de publicación más frecuente de los autores españoles en Sustainability.

Se ha determinado asimismo que prácticamente la mitad $(48,9 \%)$ de la publicación española en los números especiales coordinados por españoles corresponde a los propios editores del número o a sus colegas de universidad. Hace ya más de un siglo D. Santiago Ramón y Cajal animaba a los investigadores españoles a publicar en revistas internacionales para buscar el exequator del prestigio de los investigadores internacionales (Ramón y Cajal, 2006). Tradicionalmente, publicar en una revista internacional se asociaba a que los trabajos eran evaluados por expertos mundiales sin la subjetividad de los colegas nacionales; ahora que se demuestra que en mega-revistas con cientos de monográficos se puede dar el caso de que la mayor parte de los trabajos sean editados entre paisanos y colegas de centro, se tendrá que limitar el imaginario tradicional de lo que significaba publicar en revistas internacionales.

En relación con la temática de los artículos con firma española publicados en la revista, así como su vinculación con la materia de Sostenibilidad, se ha hallado que un porcentaje muy significativo de la producción en la revista tiene una vinculación mínima o nula con la temática de Sostenibilidad (42\%). En numerosas ocasiones las temáticas de los estudios parecen forzadas para encajar mejor en el ámbito de la Sostenibilidad. Este fenómeno es mayor en los trabajos publicados bajo el paraguas de un monográfico. En este sentido, se perciben estrategias retóricas y persuasivas de los autores para vincular en la
De los 1.417 artículos publicados por instituciones españolas en el año 2020 el $81,6 \%$ se han publicado en algún número monográfico (1.156 artículos). 194 trabajos son autoría de los propios editores de los monográficos (26,6\%). A ellos deben sumarse 163 trabajos $(22,3 \%)$ que son editados por colegas de universidad de los editores de los monográficos 
mayor medida posible su temática de investigación con el scope de la revista. Esto se produce principalmente en artículos que provienen de las Ciencias Sociales, especialmente de Educación, Economía, Deporte, y Turismo. Para superar esta falta de adecuación a la temática de la revista, se recurre a diversos elementos, por ejemplo, la alusión a los Objetivos de Desarrollo Sostenible (ODS) que son utilizados para justificar prácticamente cualquier temática. Del mismo modo, se produce un abuso del propio término "sostenibilidad" en los títulos de los trabajos, detectándose la raíz sustain* en el $26,8 \%$ de los artículos (380 trabajos), muchas veces de manera artificial. En otros casos, apenas se puede establecer vinculación alguna entre el título del trabajo y la temática de sostenibilidad. En el cuadro 2 se ofrecen ejemplos ilustrativos de estas prácticas.

Cuadro 2. Ejemplos de títulos de artículos editados en Sustainability en 2020 con vinculación nula o mínima con la materia de Sostenibilidad

\begin{tabular}{|c|c|}
\hline ODS & $\begin{array}{l}\text { Spanish archaeological museums during Covid-19 (2020): An edu-communicative analysis of their activity on Twitter through } \\
\text { the sustainable development goals }\end{array}$ \\
\hline ODS & $\begin{array}{l}\text { Massive open online courses (MOOC) within the framework of international developmental cooperation as a strategy to } \\
\text { achieve sustainable development goals }\end{array}$ \\
\hline ODS & Risks of banking services' digitalization: The practice of diversification and sustainable development goals \\
\hline Sustain* & $\begin{array}{l}\text { Developing a regression model of cooperative learning methodology in pre-service teacher education: A sustainable path for } \\
\text { transition to teaching profession }\end{array}$ \\
\hline Sustain* & $\begin{array}{l}\text { Sustainable orientation of management capability and innovative performance: The mediating effect of knowledge manage- } \\
\text { ment }\end{array}$ \\
\hline Sustain* & $\begin{array}{l}\text { Ambidextrous knowledge and learning capability: The magic potion for employee creativity and sustainable innovation } \\
\text { performance }\end{array}$ \\
\hline Sustain* & Management accounting systems, top management teams, and sustainable knowledge acquisition: Effects on performance \\
\hline Sin relación & The pavilions at the Alhambra's Court of the Lions: Graphic analysis of muqarnas \\
\hline Sin relación & Segmenting the older resident's perception of a major cycling event \\
\hline Sin relación & Measurement of service quality in trade fair organization \\
\hline Sin relación & Changes in tongue kissing in hook-ups after Covid-19 \\
\hline Sin relación & Analyzing the online reputation and positioning of airlines \\
\hline
\end{tabular}

Estos datos ponen de manifiesto la muy laxa revisión editorial que realiza la revista respecto a las temáticas de publicación. Ha de tenerse en cuenta que una de las labores del editor de la revista es velar por que los manuscritos recibidos se adecuen al alcance de la revista (Baiget, 2020), labor que parece soslayarse en Sustainability. Una posible conjetura para explicar este hecho tendría que ver con el interés comercial de la revista en abrir el abanico temático lo máximo posible, a fin de maximizar el número potencial de investigadores interesados en publicar en sus páginas. Recordemos que, al tratarse de una revista de publicación exclusivamente electrónica, no cuenta con restricciones espaciales, y que su cuenta de resultados depende del número total de artículos (de pago) publicados. Se demuestra también que esta apertura temática de la revista se da con posterioridad a la indexación en Web of Science, pues la tasa de trabajos externos a la temática era mínima previamente a su selección por el producto de Clarivate, y que este fenómeno se da en mayor medida en los números especiales que en los números regulares.

Respecto al tercer objeto específico del trabajo (Estudiar los tiempos de publicación de los trabajos españoles según su pertenencia a algunos de los subgrupos), se ha hallado que estos tiempos son algo más cortos para los trabajos publicados por el propio editor del monográfico, o por los autores de su entorno. Sin embargo, el proceso editorial es tan eficiente en todos los subgrupos analizados, que las diferencias en días (pese a que son significativas según el análisis de la Anova) son mínimas, por lo que no tiene implicaciones prácticas para los autores de uno u otro subgrupo. Otra cuestión a determinar en futuros trabajos es si una revista especializada en Sostenibilidad que publica artículos de otros temas, puede garantizar revisores que sean realmente "pares" de los autores. Igualmente, dado lo asombroso de los períodos de revisión, cabría preguntarse si es posible realizar revisiones de calidad con unos plazos tan férreamente delimitados.

\subsubsection{Implicaciones para las políticas públicas}

Estos resultados tienen unas importantes implicaciones para las políticas públicas.

La primera de ellas es la constatación de que una parte de los fondos de investigación de los que disponen las universidades españolas y sus grupos de investigación se está destinando a sufragar los APC de revistas como Sustainability, o IJERPH (cuyo APC es incluso más elevado que el de Sustainability, 2.300 francos suizos, es decir, unos 2.100 euros). Esto 
puede además afectar a los investigadores que no disponen de fondos para cubrir los costes de publicación, que deben renunciar a publicar en estos medios (salvo que les "inviten" a ser editores de monográficos, o un colega les "convide" al artículo), y acudir a revistas que no cobren por publicación. De esta manera, supone un detrimento en sus carreras profesionales ya que las revistas tradicionales cuentan con unos plazos de revisión y publicación más elevados, además de un distinto nivel de exigencia en las revisiones editoriales y en los procesos de peer review. Esto crea una evidente desigualdad entre los investigadores con fondos para APC y los que no disponen de ello, y que se extiende a otras editoriales que aplican costes de procesamiento de artículos.

Una segunda derivada se refiere a los criterios de valoración de la actividad investigadora aplicados en España, que han exacerbado la necesidad de realizar publicaciones en revistas que cumplan ciertos criterios bibliométricos, aunque éstas no sean del área específica del investigador. El denominado "efecto Aneca" (Masip, 2011) en España propicia que las revistas elegidas para la difusión de los trabajos lo sean por cómo podrían ser evaluadas en convocatorias antes que por características esenciales como su temática o audiencia. Esta adaptación de los evaluados a los criterios bajo los cuales se les mide es una constante en la evaluación de la ciencia en todo el mundo y se ha constatado para países como Australia, Reino Unido, o Polonia (Butler, 2003; Moed, 2008; Korytkowski; Kulczycki, 2019), y también en España, no siempre con efectos negativos (Jiménez-Contreras; De-Moya-Anegón; Delgado-López-Cózar, 2003).

En el caso de Sustainability, sí que parece incidir directamente en los procesos de generación y difusión del conocimiento, pudiendo acarrear consecuencias negativas para las revistas disciplinares, o con plazos de publicación más elevados. Los incentivos de publicar en las revistas emblemáticas de una disciplina se ven eclipsados si estos trabajos se pueden publicar sin competencia y en tiempo récord en medios como Sustainability que cumplen los requisitos bibliométricos demandados por las agencias. Este hecho es otro argumento para hacer a las agencias replantearse sus criterios de evaluación, pasando a un escenario más cercano a las tendencias planteadas por las responsible metrics, que proponen (entre otras cosas) la valoración de la investigación según sus contenidos, y no según el medio a través del cual se difunde (Hicks et al., 2015; DORA, 2012). Recientes acciones como la firma por parte de la Agencia Estatal de Investigación de la declaración DORA o llamamientos como el de Delgado-López-Cózar, Ràfols y Abadal (2021) por un cambio radical en la evaluación de la investigación en España ponen de manifiesto que se trata de una cuestión capital en el ecosistema investigador. Esto se hace especialmente patente en megajournals como Sustainability, donde a diferencia de las revistas clásicas no existe competición por publicar y la dependencia económica de la editorial sobre la aceptación de los manuscritos genera un claro conflicto de interés que parece no estar bien resuelto. Si hay consenso en la comunidad bibliométrica en que es incorrecto atribuir el impacto de una revista a cada uno de los trabajos que publica, esta es una realidad a gritos en revistas que publican miles de artículos en cientos de monográfico, sobre temáticas muy diversas y con niveles de calidad muy dispares. Si la evaluación mediante el Factor de Impacto a los artículos publicados en las revistas disciplinares tiene poco sentido, en las mega-revistas es complemente disparatado. El Factor de Impacto representa al conjunto de artículos cuando estos son homogéneos. En una revista clásica los grupos son homogéneos en el sentido en que se derivan de un mismo tema y son evaluados por un mismo equipo editorial, en las megajournals esto no sucede y hay variaciones grandes por áreas (Repiso; Moreno-Delgado; Torres-Salinas, 2020). Por tanto, en estas macro-revistas el Factor de Impacto es aún menos representativo que en las revistas tradicionales.

\subsubsection{Limitaciones y prospectiva}

Este trabajo aborda un caso de estudio de una única revista, un único año y un sólo país, por lo que los resultados no pueden ser extrapolados de forma directa a la situación en otros países, o incluso en otras revistas de la misma editorial, o de otras con prácticas editoriales similares. En todo caso, esta investigación pone de relieve una serie de prácticas editoriales que afecta a un número insólito de trabajos y que puede estar afectando a los patrones comunicativos de los investigadores españoles, y a sus estrategias de desarrollo profesional. En este sentido, se abren diferentes líneas de investigación sobre las que cabría profundizar desde el ámbito de la bibliometría y la evaluación de la ciencia, entre ellas:

- el rol, método de selección y conocimiento de los revisores de estas revistas;

- el prestigio y solvencia científica de los editores de los números monográficos;

- el impacto, tanto académico como social de las investigaciones publicadas en esta revista;

- la incidencia de la irrupción de este y otros medios que son en la práctica multidisciplinares en ámbitos concretos de las Ingenierías, las Ciencias de la Salud, las Ciencias Sociales, o incluso las Humanidades.

Por supuesto, también cabría investigar las estrategias editoriales en otras revistas del grupo MDPI, las de revistas de otras editoriales con prácticas similares (como Frontiers), o las de mega-revistas como Scientific reports, o IEEE access, entre otras; así como la calidad metodo-
Se tendrá que limitar el imaginario tradicional de lo que significaba publicar en revistas internacionales 
lógica de las investigaciones publicadas en estas revistas en comparación con las revistas tradicionales de cada disciplina.

Otro aspecto que debería estudiarse a futuro y que es factible es la calidad y profundidad de las revisiones. Es factible porque algunos trabajos permiten hacer público este elemento y debería estudiarse porque en el marco de esta investigación se ha accedido a unas pocas revisiones de la muestra estudiada y se encontraron evaluaciones superficiales, con propuesta siempre de cambios menores, más de carácter formal que de contenido, sin abordar aspectos específicos de la temática del trabajo. Una conjetura de verificarse este hecho podría ser que la editorial no busca "pares" reales, es decir, investigadores especialistas en la temática abordada en el manuscrito, sino revisores veloces, alentados quizá por conseguir un "ticket descuento" para su próximo artículo en MDPI.

\subsection{Conclusiones}

Se han expuesto una serie de resultados preocupantes desde el punto de vista de la comunicación científica:

- En primer lugar, la aceptación de artículos que nada tienen que ver con la temática de la revista es un elemento que afecta a la credibilidad de sus procesos de validación, pues este es el primer y fundamental elemento que se valora en el proceso de revisión editorial al recibir un manuscrito; este hecho en un número tan elevado es crítico.

- En segundo lugar, es llamativo que el modelo de publicaciones sea el de números especiales, y que la mayor parte de los investigadores españoles publiquen en monográficos editados por autores también españoles; más preocupante es que haya un elevado número de trabajos de los propios colegas de universidad de los editores.

- En último lugar los velocísimos tiempos desde el envío del manuscrito a la publicación pueden estar asociados a una reducción de la calidad del proceso.

Es necesario seguir monitorizando la actividad científica en la revista Sustainability así como en otras de la editorial MDPI (como IJERPH) para poder determinar hasta qué punto la aparición de este tipo de mega-revistas está configurando las estrategias de publicación de los académicos en España, y cómo esta modificación de las agendas de investigación está repercutiendo en las revistas disciplinares y en las propias temáticas y líneas de trabajo abordadas. En la práctica, Sustainability está operando como una mega-revista multidisciplinar fuera del ámbito de Medioambiente, afectando a la producción científica española en varias áreas.

Finalmente, los resultados derivados de este trabajo invitan a una reflexión profunda sobre el modo en que se evalúa la investigación, y en especial la publicada en revistas como la aquí analizada. El hecho de que se hayan detectado varios editores que en un solo año dirigen hasta cuatro de estos números especiales, o de que algunos autores puedan publicar más de una decena de trabajos en un solo año nos hace creer que los méritos derivados de publicar o ser editor invitado en esta revista ( $y$ en otras similares) deben ser revisados. El hallazgo de que la mayor parte de los trabajos de investigadores españoles son publicados en monográficos de paisanos, y de que un número elevado además son de los mismos editores del número o de colegas de universidad, vincula más a estas revistas con la colaboración intrainstitucional que con la internacional, recordando que para muchos esta colaboración intrainstitucional es un eufemismo para la endogamia. Por ello, es urgente que, en vistas a las evaluaciones del personal académico, estos trabajos sean valorados por su mérito individual, de acuerdo con la filosofía DORA. Está ahora mismo en debate si publicar en Sustainability es un mérito per se o en cambio se trata de un demérito costoso para las arcas de investigación españolas. Es por ello, por lo que se necesitan dos cuestiones:

- un sistema evaluativo que priorice la calidad de los trabajos frente a las revistas;

- más información sobre la calidad de estas nuevas editoriales que están surgiendo al amparo del modelo de publicación APC y cuyas revistas tienen vocación de convertirse en megajournals.

\section{Notas}

1. Datos a fecha $25 / 05 / 2021$. https://www.mdpi.com/about/journals

2. Datos a fecha 25/05/2021. https://www.mdpi.com/apc\#journal-apcs

3. Datos tomados a fecha 25/05/2021. https://www.mdpi.com/journal/sustainability

\section{4. https://www.mdpi.com/editors}




\section{Referencias}

Addinsoft (2019). XLSTAT statistical and data analysis solution.

Aneca (2019). Méritos evaluables para la acreditación nacional para el acceso a los cuerpos docentes universitarios. http://www.aneca.es/Programas-de-evaluacion/Evaluacion-de-profesorado/ACADEMIA/Criterios-Diciembre-2019

Baiget, Tomàs (2020). Manual SCImago de revistas científicas. Creación, gestión y publicación. Granada: Ediciones Profesionales de la Información. ISBN: 9788412023947

https://doi.org/10.3145/manual

Beall, Jeffrey (2017). “What I learned from predatory publishers". Biochemia medica, v. 27, n. 2, pp. 273-278. https://doi.org/10.11613/BM.2017.029

Björk, Bo-Christer (2015). "Have the 'mega-journals' reached the limits to growth?”. PeerJ, v. 3. https://doi.org/10.7717/peerj.981

Björk, Bo-Christer; Catani, Paul (2016). "Peer review in megajournals compared with traditional scholarly journals: Does it make a difference?". Learned publishing, v. 29, n. 1, 9-12.

https://doi.org/10.1002/leap.1007

Butler, Linda (2003). “Explaining Australia's increased share of ISI publications - The effects of a funding formula based on publication counts". Research policy, v. 32, n. 1, pp. 143-155.

https://doi.org/10.1016/S0048-7333(02)00007-0

Conlon, Donald E.; Morgeson, Frederick P.; McNamara, Gerry; Wiseman, Robert M.; Skilton, Paul F. (2006). "From the editors: Examining the impact and role of special issue and regular journal articles in the field of management". Academy of management journal, v. 49, n. 5, pp. 857-872.

https://doi.org/10.5465/amj.2006.22798160

Crosetto, Paolo (2021). Is MDPI a predatory publisher? https://paolocrosetto.wordpress.com/author/milanphd

De-Vrieze, Jop (2018). “Open-access journal editors resign after alleged pressure to publish mediocre papers". Science, September 4.

https://doi.org/10.1126/science.aav3129

Delgado-López-Cózar, Emilio; Ràfols, Ismael; Abadal, Ernest (2021). “Letter: A call for a radical change in research evaluation in Spain". Profesional de la información, v. 30, n. 3, e300309.

https://doi.org/10.3145/epi.2021.may.09

DORA (2012). Declaration on Research Assessment (DORA).

https://sfdora.org

Gardner, William L.; Lowe, Kevin B.; Moss, Todd W.; Mahoney, Kevin T.; Cogliser, Claudia C. (2010). "Scholarly leadership of the study of leadership: A review of The leadership quarterly's second decade, 2000-2009". The leadership quarterly, v. 21, n. 6, pp. 922-958. https://doi.org/10.1016/j.leaqua.2010.10.003

Gjengedal, Kjerstin (2019). "Publisering: Stort forlag med lite kvalitetskontroll”. Forkskerforum, 28 oktober. https://www.forskerforum.no/stort-forlag-med-lite-kvalitetskontroll

González-Sala, Francisco; Osca-Lluch, Julia; Tortosa-Gil, Francisco; Peñaranda-Ortega, María (2017). "Characteristics of monographic special issues in Ibero-American psychology journals: visibility and relevance for authors and publishers". Scientometrics, v. 112, n. 2, pp. 1069-1077.

https://doi.org/10.1007/s11192-017-2372-4

Hicks, Diana; Wouters, Paul; Waltman, Ludo; De-Rijcke, Sarah; Ràfols, Ismael (2015). “Bibliometrics: the Leiden Manifesto for research metrics". Nature, v. 520, n. 7548, pp. 429-431.

https://doi.org/10.1038/520429a

Jiménez-Contreras, Evaristo; De-Moya-Anegón, Félix; Delgado-López-Cózar, Emilio (2003). “The evolution of research activity in Spain: the impact of the National Commission for the Evaluation of Research Activity (Cneai)". Research policy, v. 32, n. 1, pp. 123-142. https://doi.org/10.1016/S0048-7333(02)00008-2

Khan, Muhammad-Asif; Ali, Imtiaz; Ashraf, Rohail (2020). "A bibliometric review of the special issues of psychology \& marketing: 1984-2020". Psychology \& marketing, v. 37, n. 9, pp. 1144-1170.

https://doi.org/10.1002/mar.21393 
Khan, Muhammad-Asif; Pattnaik, Debidutta; Ashraf, Rohail; Ali, Imtiaz; Kumar, Satish; Donthu, Naveen (2021). "Value of special issues in the journal of business research: A bibliometric analysis". Journal of business research, v. 125, pp. 295-313.

https://doi.org/10.1016/j.jbusres.2020.12.015

Korytkowski, Przemysław; Kulczycki, Emanuel (2019). “Examining how country-level science policy shapes publication patterns: The case of Poland". Scientometrics, v. 119, n. 3, pp. 1519-1543.

https://doi.org/10.1007/s11192-019-03092-1

Masip, Pere (2011). "Efecto Aneca: producción española en comunicación en el Social Sciences Citation Index". Anuario ThinkEPI, v. 5, pp. 206-210.

https://recyt.fecyt.es/index.php/ThinkEPI/article/view/30505

MDPI (2018). "Towards the multilingual web of data". [Special issue flyer]. https://www.mdpi.com/journal/information/special_issue_flyer_pdf/Web_Data/web

Moed, Henk F. (2008). “UK research assessment exercises: Informed judgments on research quality or quantity?”. Scientometrics, v. 74, n. 1, pp. 153-161.

https://doi.org/10.1007/s11192-008-0108-1

Mowday, Richard T. (2006). "If special issues of journals are not so special, why has their use proliferated?". Journal of management inquiry, v. 15, n. 4, pp. 389-393.

https://doi.org/10.1177/1056492606294639

Murray, Dan G.; Chabot, Christian (2013). Tableau your data!: Fast and easy visual analysis with Tableau software. Wiley \& Sons. ISBN: 9781118612040

Olk, Paul; Griffith, Terri L. (2004). "Creating and disseminating knowledge among organizational scholars: The role of special issues". Organization science, v. 15, n. 1.

https://doi.org/10.1287/orsc.1030.0055

Ramón y Cajal, Santiago (2006). Los tónicos de la voluntad: Reglas y consejos sobre investigación científica. Gadir Editorial. ISBN: 9788493443979

Repiso, Rafael; Moreno-Delgado, Alicia; Torres-Salinas, Daniel (2020). “If PLoS one were really 101 different specialized journals: A proposed approach to the evaluation of multidisciplinary megajournals". Learned publishing, v. 33, n. 2, pp. 96-103.

https://doi.org/10.1002/leap.1264

Repiso, Rafael; Tur-Viñes, Victoria; Segarra-Saavedra, Jesús; Hidalgo-Marí, Tatiana (2021). "The prevalence and impact of special issues in communications journals 2015-2019”. Learned publishing, first online.

https://doi.org/10.1002/leap.1406

Sainte-Marie, Maxime; Mongeon, Philippe; Larivière, Vicent (2020). “On the topicality and research impact of special issues". Quantitative science studies, v. 1, n. 1, pp. 303-319.

https://doi.org/10.1162/qss_a_00009

Siler, Kyle (2020). "Demarcating spectrums of predatory publishing: Economic and institutional sources of academic legitimacy". Journal of the Association for Information Science and Technology, v. 71, n. 11, pp. 1386-1401.

https://doi.org/10.1002/asi.24339

Spezi, Valerie; Wakeling, Simon; Pinfield, Stephen; Creaser, Claire; Fry, Jenny; Willett, Peter (2017). “Open-access mega-journals: The future of scholarly communication or academic dumping ground? A review". Journal of documentation, v. 73, n. 2, pp. 263-283.

https://doi.org/10.1108/JD-06-2016-0082

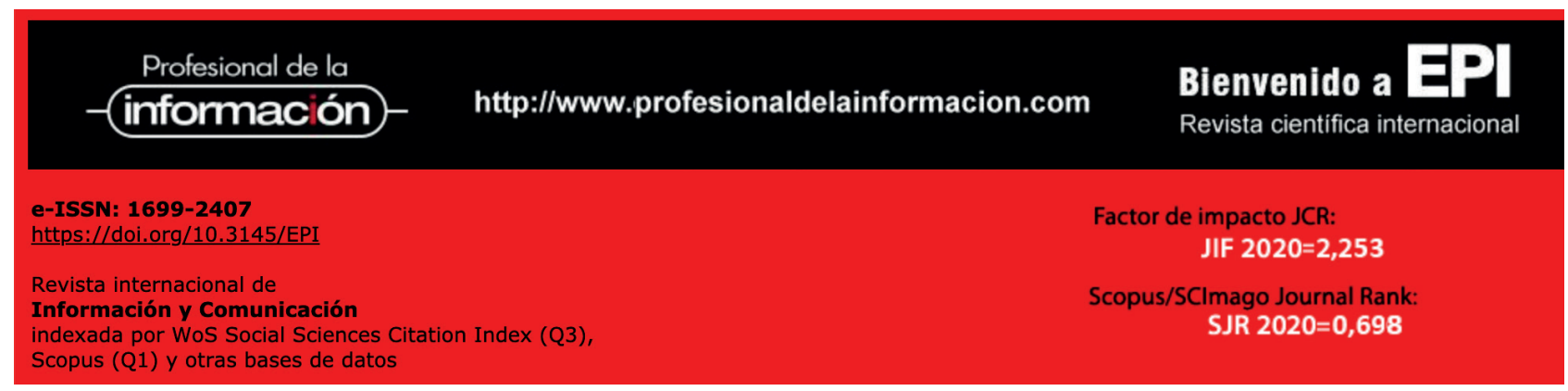

